\title{
Coenzyme Q10 serum concentration and redox status in European adults: influence of age, sex, and lipoprotein concentration
}

\author{
Petra Niklowitz, ${ }^{1, *}$ Simone Onur, ${ }^{2}$ Alexandra Fischer, ${ }^{2}$ Matthias Laudes, ${ }^{3}$ Michael Palussen, ${ }^{1}$ Thomas Menke ${ }^{1}$ \\ and Frank Döring ${ }^{2}$ \\ ${ }^{1}$ Children's Hospital of Datteln, Witten-Herdecke University, Dr.-Friedrich-Steiner-Straße 5, D-45711 Datteln, Germany \\ ${ }^{2}$ Institute of Human Nutrition and Food Science, Division of Molecular Prevention, Christian Albrechts University Kiel, \\ Heinrich-Hecht-Platz 10, 24118 Kiel, Germany \\ ${ }^{3}$ Department of Internal Medicine, University Hospital Schleswig-Holstein, Campus Kiel, Arnold-Heller-Straße 6, Haus 6, 24105 Kiel, Germany
}

(Received 11 June, 2015; Accepted 9 August, 2015; Published online 20 January, 2016)

\begin{abstract}
Coenzyme Q10 (CoQ10) is synthesized in almost all human tissues and presumably involved in age-related alterations and diseases. Here, we examined the impact of aging and sex on the serum CoQ10 status in 860 European adults ranging in age from 18 to 82 years. We identified an inverse U-shaped relationship between CoQ10 concentration and age. Women showed lower cholesteroladjusted CoQ10 levels than men, irrespective of age. As observed in both sexes, the decrease in CoQ10 concentration in older subjects was accompanied by a shift in the redox status in favour of the oxidized form. A strong positive correlation was found for total CoQ10 and cholesterol concentrations (Spearman's, $p \leq 1 \mathrm{E}-$ 74). We found strong negative correlations between total (Spearman's, $p \leq 1 \mathrm{E}-07$ ) and between cholesterol-adjusted CoQ10 concentration (Spearman's, $p \leq 1 \mathrm{E}-14$ ) and the proportion of the oxidized form of CoQ10. These correlations were not dependent on age and sex and were attenuated by supplementation with $150 \mathrm{mg} / \mathrm{day}$ reduced CoQ10 for 14 days. Overall, our results are useful to define risk groups with critical CoQ10 status in humans. In particular, older subjects were characterized by impaired CoQ10 status due to their lowered serum CoQ10 concentration and concomitant decrease of CoQ10 redox capacity.
\end{abstract}

Key Words: coenzyme Q10, ubiquinol, oxidative stress, age, sex, redox status

A geing is a multifactorial process developing at different rates depending on individual, environmental, and life style factors. ${ }^{(1)}$ Ageing is among others a substantial risk factor for most human diseases. The underlying mechanism of the aging process is still unclear. Ageing is characterized by impaired responsiveness to stress and by reduced efficacy of endogenous protective mechanisms. ${ }^{(2)}$ It is postulated that the imbalance between prooxidant generation and antioxidant defences increases during ageing. Mitochondria constitute the major source of reactive oxygen species (ROS) within the cell. The mitochondrial theory of ageing holds that the accumulation of ROS damage over time leads to age-associated mitochondrial impairment. ${ }^{(3,4)}$ Another age-related alteration is the decline in mitochondrial ability to synthesize ATP. ${ }^{(5,6)}$

Coenzyme Q10 (CoQ10) is ubiquitously synthesized in almost all cells and membranes of human tissues. CoQ10 is suspected to be involved in age-related alterations of cells and membranes. Due to its two main functions as an electron carrier in mitochondrial bioenergetics and as a lipophilic antioxidant, deficiency in CoQ10 may impair mitochondrial energy production and increase production of ROS, or susceptibility toward them. CoQ10 is associated with several age-related diseases like diabetes, hypercholesterolemia, cardiac insufficiency, and neurodegenerative diseases, and the beneficial effects of CoQ10 supplementation are discussed. ${ }^{(7,8)}$ For instance, in Parkinson's disease, there is a marked decrease in mitochondrial CoQ10 levels, ${ }^{(9)}$ while the exogenous uptake of CoQ10 slows the progressive disability. ${ }^{(10)}$

The ageing process is thought to influence CoQ concentration in tissue and plasma samples in several species. Beyer et al. ${ }^{(11)}$ found that CoQ content of several tissues in rats increased after birth, reaching a maximum level at 18 months, after which CoQ content decreased with advancing age. In pigs and mice the CoQ level decreases with age, while the oxidized proportion increases. ${ }^{(12)}$ Kamzalov et al. ${ }^{(13)}$ have postulated that age-related changes of CoQ are more obvious in mitochondria, where CoQ biosynthesis takes place. They found no significant differences in CoQ content in plasma or several tissue homogenates from rats at different ages, whereas the mitochondrial level of CoQ in tissues decreased with age. Likewise, the mitochondrial CoQ content in mouse skeletal muscle decreased with age. ${ }^{(14)}$ In rat brains, there are no changes in CoQ levels after an initial increase during the first month of life, ${ }^{(15)}$ and in human brain, ${ }^{(16,17)}$ heart and pancreas, ${ }^{(18)}$ lower concentrations of CoQ in elderly people are reported. CoQ10 measurement in tissues is considered to be the most reliable standard for diagnosis of CoQ10 deficiency.

While tissue CoQ10 levels mainly depend on de novo synthesis, ${ }^{(19)}$ plasma CoQ10 concentrations are significantly influenced by dietary uptake. ${ }^{(20)}$ However, serum or plasma CoQ10 concentrations are frequently used clinically as a raw proxy for the functional CoQ10 status in humans. CoQ10 is considered the main antioxidant in low-density lipoproteins (LDLs). Lower CoQ10 levels in plasma are related to higher lipid peroxidation. ${ }^{(21)}$ It is thought that CoQ10 levels decrease in ageing individuals. ${ }^{(22)} \mathrm{On}$ the other hand, there are studies ${ }^{(1,23,24)}$ that indicate that plasma CoQ10 levels in older people are higher than in young people. Besides ageing, sex may influence the blood level of CoQ10; given that the antioxidative defences in LDLs tend to decrease in a sex-specific manner in ageing men. ${ }^{(25)}$ As the findings on CoQ10 plasma levels during ageing are inconsistent and often not associated with lipoprotein concentration, and furthermore, information on changes in the redox status is missing, the present study was conducted to examine the impact of aging and sex on total and cholesterol-adjusted CoQ10 concentration and redox-status in a large cohort of 860 subjects aged $18-82$ years.

*To whom correspondence should be addressed.

E-mail: forschungslabor@kinderklinik-datteln.de; pf-niklowitz@web.de 


\section{Materials and Methods}

Basic study population. Sample characteristics of subjects and study design have been recently described. ${ }^{(26)}$ In short: we determined the CoQ10 status in a large European study collective $(n=1,911)$, which was based on the PopGen control cohort ${ }^{(27)}$ and the FoCus cohort. ${ }^{(28)}$ The participants in this European study collective were recruited in cooperation with the University Hospital Schleswig-Holstein (UKSH), Kiel, Germany as healthy blood donors, or in the adiposity ambulance of UKSH. In the present study, we used sub-groups 1,3 and 4 for analysis. ${ }^{(26)}$ Out of this pool, we used 860 subjects (age range 18-82 years) who fulfilled the inclusion criteria based on questionnaires regarding prevalent diseases (diagnosed by a physician). Exclusion criteria for participation were diabetes, or hepatic, renal or gastrointestinal diseases (chronic diarrhea and inflammatory bowel diseases). Subjects with a history of apoplexy or suffering from neurological disorders (Parkinson's disease, epilepsy, essential tremor, and restless legs syndrome), cardiac insufficiency or coronary heart diseases were excluded. The upper limit for total cholesterol was set at $<6.2 \mathrm{mmol} / \mathrm{L}$ (high-risk group), and for body mass index at $<40 \mathrm{~kg} / \mathrm{m}^{2}$ (adiposity grade III, extreme). The participants had an average BMI of $26.3 \pm 4.7 \mathrm{~kg} / \mathrm{m}^{2}$ and cholesterol concentration of $4.6 \mathrm{mmol} / \mathrm{L}$. A total of $58 \%$ were female. The study was approved by the Ethics Committee of the Medical Faculty and was consistent with the Declaration of Helsinki. All volunteers gave written consent.

Intervention study. Subject characteristics and study design for the intervention study have been described previously. ${ }^{(29)}$ Fifty-three healthy male volunteers aged between 21 and 48 years received $150 \mathrm{mg} /$ day of the reduced form of CoQ10 $\left(\mathrm{Q}_{10} \mathrm{H}_{2}\right.$, ubiquinol; Kaneka Corporation, Japan) with each principal meal for 14 days. Fasting blood samples were taken before $\left(\mathrm{T}_{0}\right)$ and after $\left(\mathrm{T}_{14}\right)$ supplementation. Heparin-plasma was frozen at $-84^{\circ} \mathrm{C}$ until analysis.

Sample preparation and analysis. Blood samples were taken after an overnight fast and immediately centrifuged. Serum samples were stored at $-84^{\circ} \mathrm{C}$. The simultaneous analysis of both the oxidized (ubiquinone-10) and reduced forms (ubiquinol-10) of CoQ10 was based on the method of high-performance liquid chromatography (HPLC) with electrochemical detection, as described previously. ${ }^{(30)}$ As internal standards, 56 pmol ubiquinol-9 plus 9 pmol ubiquinone-9 (Sigma-Aldrich, Taufkirchen, Germany) in $50 \mu \mathrm{l}$ ethanol were added to a $50-\mu \mathrm{l}$ serum aliquot. After hexane extraction and centrifugation $\left(5 \mathrm{~min}, 1,000 \mathrm{~g}, 4^{\circ} \mathrm{C}\right)$, the separated hexane phase was evaporated to dryness under a stream of argon and the dry residue was re-dissolved in $50 \mu \mathrm{l}$ ethanol for injection into the HPLC system. The analytical column was a Prontosil 1203-C18-SH PEEK column (Bischoff, Leonberg, Germany). The detection system consisted of a Coulochem II electrochemical detector (ESA, Bedford, MA) connected with a Model 5021A conditioning cell and a Model 5011A analytical cell. A 10- $\mu 1$ sample was stored at $-84^{\circ} \mathrm{C}$ for analysis of cholesterol (CHODPAP enzymatic photometric test, Human Diganostics, Wiesbaden, Germany).

Analysis of CoQ10 was performed during 2011-2014. On each day the co-analysis of pool samples verified that redox status and concentration were maintained within control limits ( 3 sigma).

Statistics. Statistical analysis was performed using the Winstat software package (R. Fitch Software, Bad Krozingen, Germany). Data are expressed as the mean \pm SDM. To test for significant differences regarding sex, the Mann-Whitney $U$ test was used. To test for significant differences regarding CoQ10 status and cholesterol concentration between age groups, oneway-analysis of variance (Scheffe post hoc test) was used. The correlation of parameters was tested by Spearman's rank correlation. The significance level was set at $p<0.05$ for all tests.

\section{Results and Discussion}

Sex-specific changes in CoQ10 status in different age groups. The study subjects $(n=860)$ were divided into three age groups: young $(18-40$ years, $n=326)$, middle aged (41-60 years, $n=388)$, and old (61-82 years, $n=146)$ (Table 1$)$. We found an inverse U-shaped relationship between CoQ10 concentration and age, with the highest concentrations in middle-aged subjects. The cholesterol level increased during ageing in women; whereas in men, cholesterol concentration was lower in old than middle-aged subjects. Cholesterol-related CoQ10 concentration exhibited an inverse U-shaped course during ageing, with the highest concentration in the middle-aged group without reaching statistical significance. The distinctly elevated cholesterol-related CoQ10 concentration in men compared to women was the most striking sex-related difference and found for all age groups.

Table 1. CoQ10 status and cholesterol level in 860 study subjects divided into age groups stratified by sex

\begin{tabular}{|c|c|c|c|c|}
\hline & $\begin{array}{c}\text { Age-range } \\
\text { number }\left(0^{x} /{ }^{2}\right)\end{array}$ & $\begin{array}{c}18-40 \\
326(129 / 197)\end{array}$ & $\begin{array}{c}41-60 \\
388(177 / 211)\end{array}$ & $\begin{array}{c}61-82 \\
146(55 / 91)\end{array}$ \\
\hline \multirow[t]{4}{*}{ CoQ10 ( $\mu \mathrm{mol} / \mathrm{L})$} & Total & $0.759 \pm 0.268^{A}$ & $0.885 \pm 0.302^{c}$ & $0.830 \pm 0.235^{\mathrm{B}}$ \\
\hline & Male & $0.796 \pm 0.293^{A}$ & $0.939 \pm 0.335^{\mathrm{B}}$ & $0.835 \pm 0.235^{A B}$ \\
\hline & Female & $0.734 \pm 0.248^{A}$ & $0.839 \pm 0.265^{\mathrm{B}}$ & $0.827 \pm 0.236^{\mathrm{B}}$ \\
\hline & $p \leq\left(\sigma^{\top}\right.$ vs $\left.q\right)$ & & 0.01 & \\
\hline \multirow[t]{4}{*}{ Cholesterol (mmol/L) } & Total & $4.33 \pm 0.79^{A}$ & $4.78 \pm 0.73^{B}$ & $4.80 \pm 0.68^{B}$ \\
\hline & Male & $4.27 \pm 0.88^{A}$ & $4.83 \pm 0.74^{\mathrm{B}}$ & $4.53 \pm 0.60^{A}$ \\
\hline & Female & $4.37 \pm 0.72^{\mathrm{A}}$ & $4.74 \pm 0.71^{\mathrm{B}}$ & $4.97 \pm 0.67^{c}$ \\
\hline & $p \leq\left(\sigma^{\top}\right.$ vs $\left.\rho^{\prime}\right)$ & & & 0.001 \\
\hline \multirow{4}{*}{$\begin{array}{l}\text { CoQ10/cholesterol } \\
(\mu \mathrm{mol} / \mathrm{mol})\end{array}$} & Total & $175 \pm 53^{A}$ & $184 \pm 53^{A}$ & $173 \pm 44^{\mathrm{A}}$ \\
\hline & Male & $185 \pm 51^{\mathrm{A}}$ & $192 \pm 53^{A}$ & $184 \pm 46^{A}$ \\
\hline & Female & $169 \pm 54^{\mathrm{A}}$ & $177 \pm 53^{A}$ & $166 \pm 42^{\mathrm{A}}$ \\
\hline & $p \leq\left(\sigma^{\top}\right.$ vs o $)$ & 0.005 & 0.005 & 0.01 \\
\hline \multirow{4}{*}{$\begin{array}{l}\text { Redox-status } \\
\text { (\% oxidized in total) }\end{array}$} & Total & $12.7 \pm 2.4^{\mathrm{A}}$ & $12.8 \pm 2.2^{\mathrm{A}}$ & $13.7 \pm 2.0^{\mathrm{B}}$ \\
\hline & Male & $12.4 \pm 2.7^{\mathrm{A}}$ & $12.5 \pm 2.0^{A}$ & $13.7 \pm 2.3^{B}$ \\
\hline & Female & $12.9 \pm 2.1^{\mathrm{A}}$ & $13.0 \pm 2.4^{\mathrm{A}}$ & $13.6 \pm 1.9^{B}$ \\
\hline & $p \leq\left(\sigma^{7}\right.$ vs $\left.{ }^{\prime}\right)$ & 0.005 & & \\
\hline
\end{tabular}

Data are shown as mean \pm SDM; data with different superscripts are statistically different in regard to age groups as calculated by ANOVA (Scheffe post-hoc test) $p \leq 0.05$; sex-related differences within age groups were calculated by Mann-Whitney $U$ test. 
Compared to the young and middle-aged groups, the proportion of the oxidized form of CoQ10 was higher in older individuals in both sexes. In young subjects (18-40 years) the proportion of oxidized CoQ10 was higher in women than men. The oxidized proportion of CoQ10 was high in the present study (mean $12.9 \%$ ). Our own previous studies yielded mean oxidized proportions of CoQ10 in healthy children and adults of $\sim 9 \%,{ }^{(31,32)}$ however, the redox status of CoQ10 was analysed in heparin plasma in these studies. A comparative analysis of intra-day variations of serum versus heparin plasma pool revealed significantly increased oxidized proportions in serum, while the total concentration was unaffected (data not shown). However, this did not influence sex differences or the general age course of the redox status.

The redox status of CoQ10 is suggested to be a useful biomarker of oxidative stress. ${ }^{(33)}$ In its reduced form, CoQ10 is one of the most potent endogenously synthesized lipophilic antioxidants. $^{(34)} \mathrm{A}$ shift towards oxidized CoQ10 is likely a sign of increased oxidative stress. ${ }^{(35-37)}$ High levels of CoQ10 in lipoproteins are directly related to high resistance to initiation of lipid peroxidation. $^{(38)}$ Since men had increased cholesterol-related concentrations combined with increased total ubiquinol concentrations, the antioxidative capacity and demand seemed to be sex specific.

Sex-related differences were also found by Del Pozo-Cruz et al. ${ }^{(24)}$ who showed that elderly men had greater CoQ10/cholesterol ratios than elderly women; however, men also exhibited higher lipid peroxidation, which suggests an increased demand in antioxidant capacity in old men. That study group also found that BMI did not influence the lipid profile but showed a negative correlation with total CoQ10 level, which contradicts our own findings. We found a positive association between BMI and cholesterol concentration, as well as between BMI and both CoQ10 concentration and oxidized proportion. However, the upper inclusion limits for BMI and cholesterol concentration were set at higher level in our study.

Kaikkonen et al. ${ }^{(23)}$ found a positive association between total plasma CoQ10 and age, while men had higher levels than women ( $n=518$; age range 45-70 years). Lu and Frank ${ }^{(39)}$ found no significant differences in CoQ10 concentration with respect to sex but an age-dependent increase in total CoQ10 concentration $(n=1,317$; age range 1-94 years). Unfortunately, in both studies, no information was given about lipid-adjusted CoQ10 or redox status. Miles et $a l^{(40)}$ found in the plasma of self-reported healthy adults ( $n=148$; age range $28-80$ years) significantly increased CoQ10 concentration in men compared to women. However, this sexrelated difference equalized, when CoQ10 concentration was related to cholesterol. They also reported that the redox status of CoQ10 in plasma was independent of sex. This contradicts our own findings in which sex-related differences in CoQ10 concentration were strengthened after lipid adjustment, and the oxidized proportion of CoQ10 was higher in young women compared to young men. Differences in the analysis of CoQ10 in plasma between the study groups may be explained by factors like race differences or divergent life habits.

In the present study, the cholesterol-related CoQ10 concentrations of adults showed distinctly lower values than those previously found for healthy children, ${ }^{(31)}$ in which an age-related decrease during childhood was evident: while infants $(n=85,0-1$ year) had mean values of $303 \mu \mathrm{mol} \mathrm{CoQ10/mol} \mathrm{cholesterol,} \mathrm{there}$ was a significant decrease to 260 in preschool children $(n=60,2-$ 6 years) and to 226 in school-aged children ( $n=54,6-15$ years). This decrease in lipid-adjusted CoQ10 concentrations during childhood was confirmed by others. ${ }^{(41)}$ Likewise, there was a decrease in total CoQ10 during childhood, reaching the level of young adults during school age (mean $0.807 \mu \mathrm{mol}$ CoQ10/1). ${ }^{(31)}$ However, during adulthood the total CoQ10 concentration in blood again increases with age with a peak at 41-60 years, and decreases in old age, especially in men.

Relationships between CoQ10 concentration and total cholesterol, LDL cholesterol, HDL cholesterol, and BMI. As shown in Table 2, there was a strong positive correlation between CoQ10 and cholesterol concentrations. Concentrations of both parameters showed similar and parallel changes with age (Fig. 1). CoQ10 and LDL-cholesterol concentrations also showed a strong positive correlation (Table 2). In contrast, HDL-cholesterol level was not significantly related to CoQ10 concentration. The strong relationship between the total cholesterol or the LDL-cholesterol and CoQ10 concentration respectively in serum was anticipated as virtually all CoQ10 in the circulation is associated with lipoproteins. ${ }^{(42)}$ Furthermore, CoQ10 and cholesterol share in parts a common synthetic pathway. ${ }^{(2)}$ However, the present findings emphasize the necessity for adjustment of CoQ10 concentration to lipid concentration while diagnosing the functional status of CoQ10 in human blood.

Both CoQ10 and cholesterol concentrations were positively correlated with BMI (Table 2). BMI and the proportion of the oxidized form of CoQ10 were also positively correlated (Table 2), which indicates increased oxidative stress in obese people.

Correlation between CoQ10 concentration and redox state. We found a strong negative correlation (Spearman $p \leq 1 \mathrm{E}-07)$ between the concentration of CoQ10 and the proportion of the oxidized form of CoQ10 (Table 2). This correlation was even stronger after correction of CoQ10 concentration for cholesterol concentration. The resulting negative correlation between CoQ10/cholesterol and redox state of CoQ10 reached Spearman values of $p \leq 1 \mathrm{E}-14$ (Table 2 ). This correlation was present in all age groups. Namely, subjects with a low concentration of CoQ10 had a concomitant reduced redox capacity of CoQ10, meaning less ubiquinol and more ubiquinone. Based on these results, all data points were stratified into four groups, and compartmented into quadrants corresponding to mean values of both cholesteroladjusted CoQ10 concentration and redox state of CoQ10 (Fig. 2). Of special interest are group I (low concentration, high oxidized form) and group IV (high concentration, low oxidized form), representing a low (group I) and high (group IV) CoQ10 status, respectively. Forty-one percent of all subjects in the old-age group

Table 2. Correlation (Spearman's rank correlation) of parameters of CoQ10 status, cholesterol concentration, and BMI in 860 study subjects ranging in age from 18 to 82 years

\begin{tabular}{llcc}
\hline Parameter 1 & Parameter 2 & $p \leq$ & Correlation \\
\hline Total CoQ10 & Total cholesterol & $1.00 \mathrm{E}-74$ & 0.57 \\
Total CoQ10 & HDL-cholesterol & $\mathrm{ns}$ & 0.54 \\
Total CoQ10 & LDL-cholesterol & $1.00 \mathrm{E}-30$ & 0.13 \\
Total CoQ10 & BMI & $1.00 \mathrm{E}-04$ & 0.12 \\
Total cholesterol & BMI & 0.001 & 0.10 \\
Redox-status (\% oxidized CoQ10) & BMI & 0.002 & -0.18 \\
Redox-status (\% oxidized CoQ10) & Total CoQ10 & $1.00 \mathrm{E}-07$ & -0.27 \\
Redox-status (\% oxidized CoQ10) & CoQ10/cholesterol & $1.00 \mathrm{E}-14$ & \\
\hline
\end{tabular}




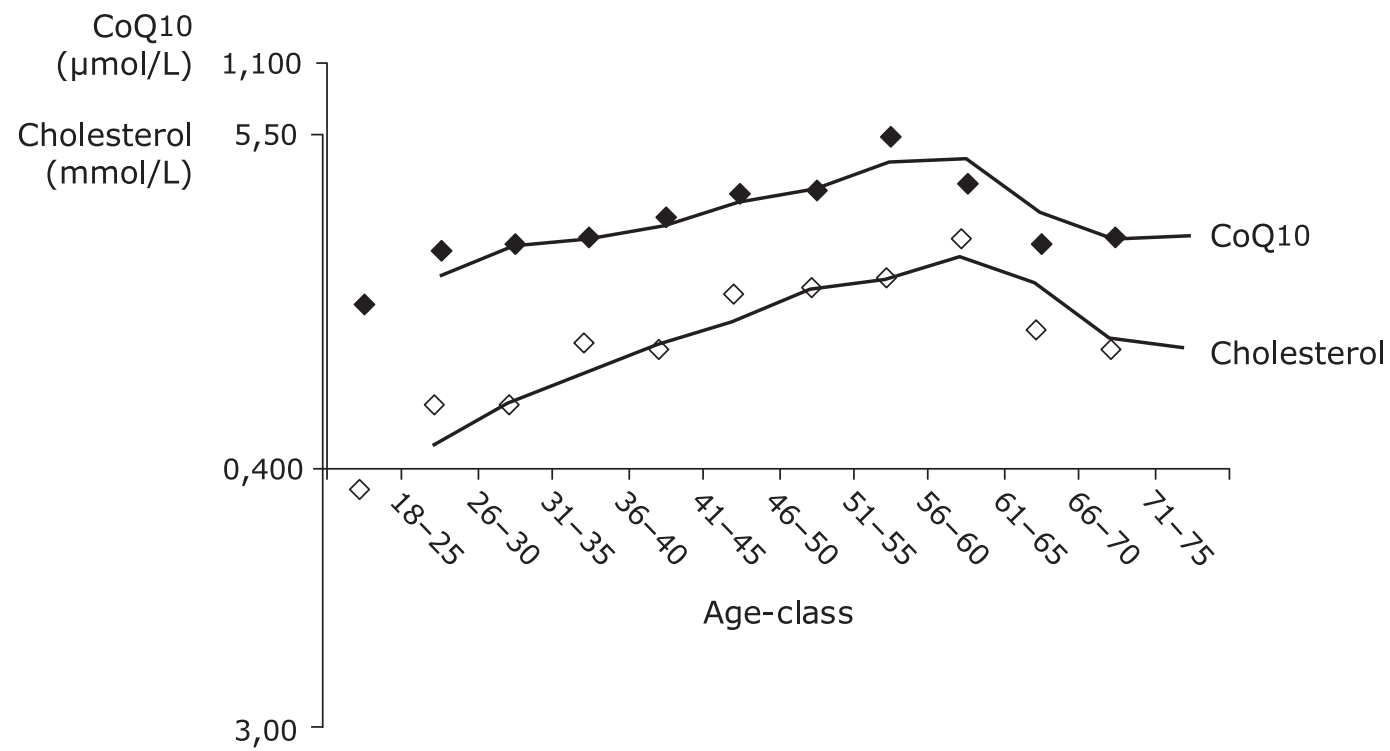

Fig. 1. CoQ10 and cholesterol concentrations of 860 subjects split into age-groups (5-year-steps). Mean values are connected by trend lines of moving average.

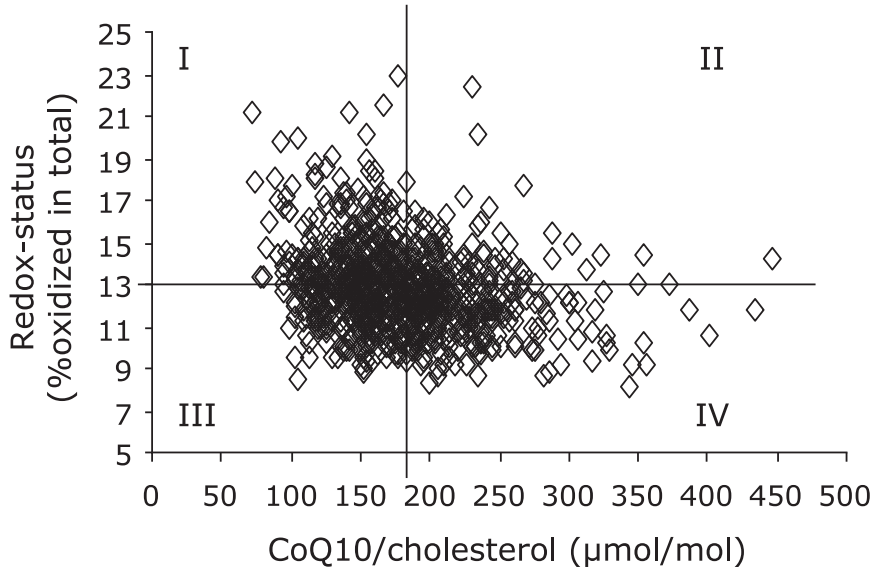

Fig. 2. Cholesterol-adjusted CoQ10 concentration correlated to redox status of CoQ10 (Spearman's, $p \leq 1 \mathrm{E}-14$ ) in 860 subjects ranging in age from 18 to 82 years: compartmented into quadrants corresponding to mean values of all data points $(12.9 \%$ redox status, $179 \mu \mathrm{mol}$ CoQ10/mol cholesterol).

(61-82 years) comprised group I, but only $19 \%$ group IV. Subjects with low total CoQ10 in the circulation are suspected of having impaired antioxidative capacity, an increased consumption of their antioxidative resources, or to be more susceptible towards oxidative stress. This situation is intensified when the amount of the antioxidant CoQ10 is not able to assure the protection of the lipoproteins. Since subjects in the old age group (61-82 years) showed increased oxidized proportions of CoQ10 combined with decreasing levels in total CoQ10, elderly people may be more susceptible towards oxidative stress. However, future studies should also consider the association between CoQ10 status, age and other biomarkers for oxidative stress, such as glutathione Stransferase or malondialdehyde levels. It was shown by Del PozoCruz et al..$^{(24)}$ that physical activity in individuals of advanced age increased the levels of total and lipid-adjusted CoQ10, and lowered the levels of lipid peroxidation in human plasma.

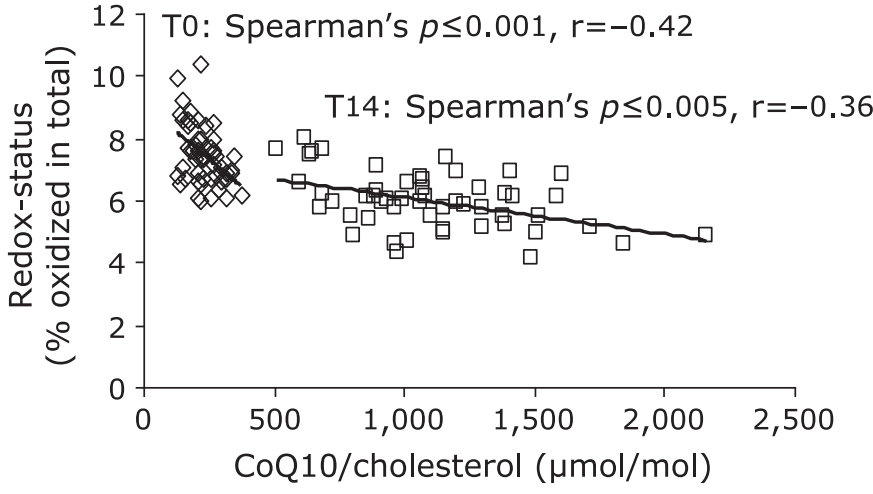

Fig. 3. Correlation of cholesterol-adjusted CoQ10 concentration and redox status in 53 male volunteers before (T0) and after 14 days (T14) of supplementation with CoQ10 (150 mg ubiquinol/day).

However, a reliable improvement in antioxidant capacity may be achieved by oral supplementation of CoQ10. Re-analysis of our intervention study ${ }^{(29)}$ revealed that supplementation of $150 \mathrm{mg} /$ day ubiquinol for 14 days attenuated the negative correlation between CoQ10 concentration and redox state of CoQ10: the cholesterolrelated CoQ10 concentration significantly increased (MannWhitney $U$ test $p \leq 0)$, and the oxidized proportion of CoQ10 significantly decreased (Mann-Whitney $U$ test $p \leq 1 \mathrm{E}-10$ ) while the negative correlation between both parameters flattened (Fig. 3 ).

Conclusions. In the current study, it was shown that CoQ10 blood concentrations showed an inverse U-shaped course during adulthood. In old people, the antioxidative properties of CoQ10 in the circulation may be impaired, as reflected by a decrease in CoQ10 concentrations, accompanied by a shift in redox status in favour of the oxidized proportion. This shift in redox status was seen in both sexes; however, women irrespective of age had lower cholesterol-adjusted CoQ10 levels in comparison to men. Therefore, women may either be more susceptible to oxidative damage or men may have an increased demand in antioxidant capacity. There is a clear negative correlation between the 
cholesterol-adjusted CoQ10 level and its oxidized proportion of CoQ10. Thus, the decrease of CoQ10 levels in old age associated with a loss of antioxidant capacity is not limited to human tissues or cell organelles, but applies also to lipoprotein protection in the blood. The concurrent occurrence of low cholesterol-adjusted concentrations and high oxidized proportions of CoQ10 increase the susceptibility to oxidative stress in old age, which may be overcome by oral supplementation.

\section{Acknowledgments}

This work was supported by the patients' self-help group "Elterninitiative Tumorkranker Kinder e.V. der Vestischen Kinderklinik Datteln", Germany, and by the foundation "Peter und Ruth Wirts Stiftung", Switzerland. We thank all participants of the study cohort for their invaluable contribution to the study. The popgen 2.0 network is supported by a grant from the German

\section{References}

1 Del Pozo-Cruz J, Rodríguez-Bies E, Ballesteros-Simarro M, et al. Physical activity affects plasma coenzyme Q10 levels differently in young and old humans. Biogerentology 2014; 15: 199-211.

2 Corbi G, Conti V, Russomanno G, et al. Is physical activity able to modify oxidative damage in cardiovascular aging? Oxid Med Cell Longev 2012. DOI: $10.1155 / 2012 / 728547$

3 Harman D. Aging: a theory based on free radical and radical chemistry. $J$ Gerontol 1956; 11: 298-300.

4 Peterson CM, Johannsen DL, Ravussin E. Skeletal muscle mitochondria and aging: a review. J Aging Res 2012. DOI:10.1155/2012/194821

5 Ferguson M, Mockett RJ, Shen Y, Orr WC, Sohal RS. Age-associated decline in mitochondrial respiration and electron transport in Drosophila melanogaster. Biochem J 2005; 390 (Pt 2): 501-511.

6 Sohal RS, Forster MJ. Coenzyme Q, oxidative stress and aging. Mitochondrion 2007; 7 Suppl: S103-S111.

7 Quinzii CM, DiMauro S, Hirano M. Human coenzyme $Q_{10}$ deficiency. Neurochem Res 2007; 32: 723-727.

8 López-Lluch G, Rodríguez-Aguilera JC, Santos-Ocaña C, Navas P. Is coenzyme Q a key factor in aging?. Mech Aeging Dev 2010; 131: 225-235.

9 Shults CW, Haas RH, Passov D, Beal MF. Coenzyme Q10 levels correlate with the activities of complexes I and II/III in mitochondria from parkinsonian and nonparkinsonian subjects. Ann Neurol 1997; 42: 261-264.

10 Shults CW. Therapeutic role of coenzyme Q(10) in Parkinson's disease. Pharmacol Ther 2005; 107: 120-130.

11 Beyer RE, Burnett BA, Cartwright KJ, et al. Tissue coenzyme Q (ubiquinone) and protein concentrations over the life span of the laboratory rat. Mech Ageing Dev 1985; 32: 267-281.

12 Onur S, Niklowitz P, Fischer A, et al. A comparative study into alterations of coenzyme Q redox status in ageing pigs, mice, and worms. Biofactors 2014; 40: $346-354$.

13 Kamzalov S, Sohal RS. Effect of age and caloric restriction on coenzyme Q and alpha-tocopherol levels in the rat. Ex Gerontol 2004; 39: 1199-1205.

14 Lass A, Kwong L, Sohal RS. Mitochondrial coenzyme Q content and aging. Biofactors 1999; 9: 199-205.

15 Zhang Y, Appelkvist EL, Kristensson K, Dallner G. The lipid compositions of different regions of rat brain during development and aging. Neurobiol Aging 1996; 17: 869-875.

16 Söderberg M, Edlund C, Kristensson K, Dallner G. Lipid compositions of different regions of the human brain during aging. J Neurochem 1990; 54: 415-423.

17 Wada H, Goto H, Hagiwara S, Yamamoto Y. Redox status of coenzyme Q10 is associated with chronological age. J Am Geriatr Soc 2007; 55: 1141-1142.

18 Turunen M, Olsson J, Dallner G. Metabolism and function of coenzymeQ. Biochim Biophys Acta 2004; 1660: 171-199.

19 Hargreaves IP. Ubiquinone: cholesterol's reclusive cousin. Ann Clin Biochem 2003; 40 (Pt 3): 207-218.

20 Zhang Y, Aberg F, Appelkvist EL, Dallner G, Ernster L. Uptake of dietary coenzyme Q supplement is limited in rats. J Nutr 1995; 125: 446-453.

21 Stocker R, Bowry VW, Frei B. Ubiquinol-10 protects human low density lipoprotein more efficiently against lipid peroxidation than does alpha-
Ministry for Education and Research (01EY1103). The FoCus cohort is supported by a grant of the German Ministry for Education and Research (0315540A). Determining and analyzing the CoQ10 status in the large study population is supported by Kaneka Corporation, Japan.

\section{Abbreviations}

CoQ

HDL high-density lipoprotein

HPLC high-performance liquid chromatography

LDL low-density lipoprotein

ROS reactive oxygen species

\section{Conflict of Interest}

No potential conflicts of interest were disclosed.

Proc Natl Acad Sci US A 1991; 88: 1646-1650.

22 Ernster L, Dallner G. Biochemical, physiological and medical aspects of ubiquinone function. Biochim Biophys Acta 1995; 1271: 195-204.

23 Kaikkonen J, Nyyssönen K, Tuomainen TP, Ritonmaa U, Salonen JT. Determinants of plasma coenzyme Q10 in humans. FEBS Lett 1999; 443: 163-166.

24 Del Pozo-Cruz J, Rodríguez-Bies E, Navas-Enamorado I, Del Pozo-Cruz B, Navas P, López-Lluch G. Relationship between functional capacity and body mass index with plasma coenzyme Q10 and oxidative damage in communitydwelling elderly people. Exp Gerontol 2014; 52: 46-54.

25 Aejmelaeus R, Metsä-Ketelä T, Laippala P, Solakivi T, Alho H. Ubiquinol10 and total peroxyl radical trapping capacity of LDL lipoproteins during aging: the effects of Q-10 supplementation. Mol Aspects Med 1997; 18 Suppl: S113-S120.

26 Onur S, Niklowitz P, Fischer A, et al. Determination of the Coenzyme $\mathrm{Q}_{10}$ status in a large Caucasian study population. Biofactors 2015; 41: 211-221.

27 Krawczak M, Nikolaus S, von Eberstein H, Croucher PJ, El Mokhtari NE, Schreiber S. PopGen: population-based recruitment of patients and controls for the analysis of complex genotype-phenotype relationships. Community Genet 2006; 9: 55-61.

28 Müller N, Schulte DM, Türk K, et al. IL-6 blockade by monoclonal antibodies inhibits apolipoprotein (a) expression and lipoprotein (a) synthesis in humans. J Lipid Res 2015; 56: 1034-1042.

29 Schmelzer C, Niklowitz P, Okun JG, Haas D, Menke T, Döring F. Ubiquinol-induced gene expression signatures are translated into altered parameters of erythropoiesis and reduced low density lipoprotein cholesterol levels in humans. IUBMB Life 2011; 63: 42-48.

30 Menke T, Niklowitz P, Adam S, Weber M, Schlüter B, Andler W. Simultaneous detection of ubiquinol-10, ubiquinone-10, and tocopherols in human plasma microsamples and macrosamples as a marker of oxidative damage in neonates and infants. Anal Biochem 2000; 282: 209-217.

31 Menke T, Niklowitz P, Schlüter B, et al. Plasma levels and redox status of coenzyme Q10 in infants and children. Biofactors 2004; 20: 173-181.

32 Niklowitz P, Menke T, Andler W, Okun JG. Simultaneous analysis of coenzyme Q10 in plasma, erythrocytes and platelets: comparison of the antioxidant level in blood cells and their environment in healthy children and after oral supplementation in adults. Clin Chim Acta 2004; 342: 219-226.

33 Lagendijk J, Ubbink JB, Vermaak WJ. Measurment of the ratio between the reduced and oxidized forms of coenzyme Q10 in human plasma as a possible marker of oxidative stress. J Lipid Res 1996; 37: 67-75.

34 Bentinger M, Brismar K, Dallner G. The antioxidant role of coenzyme Q Mitochondrion 2007; 7 Suppl: S41-S50.

35 Kontush A, Reich A, Baum K, et al. Plasma ubiquinol-10 is decreased in patients with hyperlipidaemia. Atherosclerosis 1997; 129: 119-126.

36 Hara K, Yamashita S, Fujisawa A, Ishiwa S, Ogawa T, Yamamoto Y. Oxidative stress in newborn infants with and without asphyxia as measured by plasma antioxidants and free fatty acids. Biochem Biophys Res Commun 1999; 257: 244-248.

37 Sohmiya M, Tanaka M, Tak NW, et al. Redox status of plasma coenzyme Q10 indicates elevated systemic oxidative stress in Parkinson's disease. $J$ 
Neurol Sci 2004; 223: 161-166.

38 Mohr D, Bowry VW, Stocker R. Dietary supplementation with coenzyme Q10 results in increased levels of ubiquinol-10 within circulating lipoproteins and increased resistance of low-density lipoprotein to the initiation of lipid peroxidation. Biochim Biophys Acta 1992; 1126: 247-254.

39 Lu J, Frank EL. Measurement of coenzyme Q10 in clinical practice. Clin Chim Acta 2007; 384: 180-181.

40 Miles MV, Horn PS, Morrison JA, Tang PH, DeGrauw T, Pesce AJ. Plasma coenzyme Q10 reference intervals, but not redox status, are affected by gender and race in self-reported healthy adults. Clin Chim Acta 2003; 332 : $123-132$.

41 Miles MV, Horn PS, Tang PH, et al. Age-related changes in plasma coenzyme Q10 concentrations and redox state in apparently healthy children and adults. Clin Chim Acta 2004; 347: 139-144.

42 Tomasetti M, Alleva R, Solenghi MD, Littarru GP. Distribution of antioxidants among blood components and lipoproteins: significance of lipids/ CoQ10 ratio as a possible marker of increased risk for artheriosclerosis. Biofactors 1999; 9: 231-240. 